\title{
ON THE PYTKEEV PROPERTY IN SPACES OF CONTINUOUS FUNCTIONS
}

\author{
PETR SIMON AND BOAZ TSABAN \\ (Communicated by Julia Knight)
}

\begin{abstract}
Answering a question of Sakai, we show that the minimal cardinality of a set of reals $X$ such that $C_{p}(X)$ does not have the Pytkeev property is equal to the pseudo-intersection number $\mathfrak{p}$. Our approach leads to a natural characterization of the Pytkeev property of $C_{p}(X)$ by means of a covering property of $X$, and to a similar result for the Reznichenko property of $C_{p}(X)$.
\end{abstract}

\section{INTRODUCTION}

Recall that a topological space $Y$ has countable tightness if, for each $A \subseteq Y$ and each $y \in \bar{A}$, there is a countable set $B \subseteq A$ such that $y \in \bar{B}$. Pytkeev [4] has introduced the following strengthening of countable tightness.

Definition 1. A topological space $Y$ has the Pytkeev property if for each $A \subseteq Y$ and each $y \in \bar{A} \backslash A$, there exist infinite subsets $A_{1}, A_{2}, \ldots$ of $A$ such that each neighborhood of $y$ contains some $A_{n}$.

The most well studied case is where $Y=C_{p}(X)$, the collection of all continuous real-valued functions on a topological space $X$ with the topology inherited from the Tychonoff product space $\mathbb{R}^{X}$. Sakai's paper [7] is a good survey on properties of this type.

$C_{p}(X)$ is a topological group, whose identity is the constant zero function $\mathbf{0 .}$ Thus, it suffices to study the Pytkeev property and other local properties of $C_{p}(X)$ at $\mathbf{0}$ (or at any other point). The sets

$$
\left[x_{1}, \ldots, x_{n} ; k\right]=\left\{f \in C_{p}(X):\left|f\left(x_{1}\right)\right|, \ldots,\left|f\left(x_{n}\right)\right|<\frac{1}{k}\right\},
$$

where $n, k \in \mathbb{N}$ and $x_{1}, \ldots, x_{n} \in X$, form a neighborhood base at $\mathbf{0}$.

It is well known that if $X \subseteq \mathbb{R}$ is countable, then $C_{p}(X)$ has the Pytkeev property (see also Lemma 6 ). On the other hand, if $C_{p}(X)$ has the Pytkeev property and $X$ is Tychonoff, then $X$ is zero-dimensional [5]. Thus, $C_{p}(\mathbb{R})$ does not have the Pytkeev property. In his plenary talk at the Second Workshop on Coverings, Selections, and Games in Topology (Lecce, December 2005), Sakai asked what is the minimal cardinality of a set $X \subseteq \mathbb{R}$ such that $C_{p}(X)$ does not have the Pytkeev property. Theorem 3 answers this question.

Received by the editors June 20, 2006, and, in revised form, November 16, 2006.

2000 Mathematics Subject Classification. Primary 54C35, 03E17.

The second author was partially supported by the Koshland Center for Basic Research. 
Our terminology concerning combinatorial cardinals of the continuum is standard, and the topic is surveyed in [1]. The power set $\mathcal{P}(\mathbb{N})$ is identified with $\{0,1\}^{\mathbb{N}}$ using characteristic functions. This defines a topology on $\mathcal{P}(\mathbb{N})$, and the family $[\mathbb{N}]^{\aleph_{0}}=\{A \subseteq \mathbb{N}: A$ is infinite $\}$ is viewed as a subspace of $\mathcal{P}(\mathbb{N})$. As $\{0,1\}^{\mathbb{N}}$ is homeomorphic to the Cantor set $C \subseteq \mathbb{R}$ and the properties we study are topological, we may think of $\{0,1\}^{\mathbb{N}}$ and $[\mathbb{N}]^{\aleph_{0}}$ as subsets of $\mathbb{R}$.

On several occasions, we will use the following simple observation.

Lemma 2. In Definition 1, it suffices to require that for each neighborhood $U$ of $y$ there is $n$ such that $A_{n} \subseteq^{*} U$.

Proof. Assume that $A_{1}, A_{2}, \ldots$ are as in the current lemma. By moving to subsets, we may assume that each $A_{n}$ is countable. Then the family $\left\{A_{n} \backslash F: n \in \mathbb{N}, F \in\right.$ $\left.\left[A_{n}\right]^{<\aleph_{0}}\right\}$ is countable, and can therefore be enumerated as $\left\{A_{n}^{\prime}: n \in \mathbb{N}\right\}$. Clearly, $A_{1}^{\prime}, A_{2}^{\prime}, \ldots$ are as required in Definition 1 .

\section{The minimal CARdinality Where the Pytkeev Property fails}

Theorem 3. The minimal cardinality of a set $X \subseteq \mathbb{R}$ such that $C_{p}(X)$ does not have the Pytkeev property is equal to $\mathfrak{p}$.

Theorem 3 follows from several results which are of independent interest.

Recall that $\mathfrak{p}$ is the minimal cardinality of a centered family in $[\mathbb{N}]^{\aleph_{0}}$ which has no pseudo-intersection. It is easy to see that if $\mathcal{F}$ is such a family, and we close $\mathcal{F}$ under intersections of finite subsets, then for each finite partition $\mathcal{F}=\bigcup_{n=1}^{k} \mathcal{F}_{n}$, there is some $n \leq k$ such that $\mathcal{F}_{n}$ has no pseudo-intersection. The following is a natural strengthening of this Ramsey-theoretic property.

Definition 4. $\mathfrak{p}_{\sigma}$ is the minimal cardinality of a centered family $\mathcal{F} \subseteq[\mathbb{N}]^{\aleph_{0}}$ such that for each partition $\mathcal{F}=\bigcup_{n \in \mathbb{N}} \mathcal{F}_{n}$, there is $n$ such that $\mathcal{F}_{n}$ has no pseudointersection.

Remark 5. As in Lemma 2, it suffices to require in Definition 4 that there is $n$ such that $\bigcap \mathcal{F}_{n}$ is finite.

A classical result of Arkhangel'skii and Pytkeev asserts that for a Tychonoff space $X, C_{p}(X)$ has countable tightness if, and only if, all finite powers of $X$ are Lindelöf. In particular, for each $X \subseteq \mathbb{R}, C_{p}(X)$ has countable tightness.

Lemma 6. Assume that $C_{p}(X)$ has countable tightness. If $|X|<\mathfrak{p}_{\sigma}$, then $C_{p}(X)$ has the Pytkeev property.

Proof. Assume that $A \subseteq C_{p}(X)$ and $\mathbf{0} \in \bar{A} \backslash A$. As $C_{p}(X)$ has countable tightness, we may assume that $A$ is countable. The family

$$
\mathcal{F}=\left\{\left[x_{1}, \ldots, x_{n} ; k\right] \cap A: n, k \in \mathbb{N}, x_{1}, \ldots, x_{n} \in X\right\}
$$

is centered, it is a trace of a neighborhood base at $\mathbf{0}$ on $A$, and its cardinality does not exceed $|X| \cdot \aleph_{0}<\mathfrak{p}_{\sigma}$. Hence $\mathcal{F}$ can be written as $\mathcal{F}=\bigcup_{n} \mathcal{F}_{n}$, where each family $\mathcal{F}_{n}$ has a pseudo-intersection $A_{n}$. The sets $A_{n}, n \in \mathbb{N}$, witness the Pytkeev property (recall Lemma 2).

Lemma 7. There is a set $X \subseteq \mathbb{R}$ with $|X|=\mathfrak{p}_{\sigma}$, such that $C_{p}(X)$ does not have the Pytkeev property. 
Proof. Choose a centered family $\mathcal{F} \subseteq\left[\mathbb{N}^{\aleph_{0}}\right.$ of cardinality $\mathfrak{p}_{\sigma}$ in accordance with the definition of $\mathfrak{p}_{\sigma}$ : whenever $\mathcal{F}$ is expressed as a union $\bigcup_{n} \mathcal{F}_{n}$, there is some $n$ such that $\mathcal{F}_{n}$ has no pseudo-intersection. Recall from the Introduction that $[\mathbb{N}]^{\aleph_{0}}$ is viewed as a subset of $\mathbb{R}$.

For each $k$, define a mapping $g_{k}: \mathcal{F} \rightarrow \mathbb{R}$ by

$$
g_{k}(A)= \begin{cases}0 & k \in A, \\ 1 & k \notin A,\end{cases}
$$

for all $A \in \mathcal{F}$. The mapping $g_{k}$ is continuous: indeed, $g_{k}^{-1}(1-\varepsilon, 1+\varepsilon) \subseteq$ $\left\{x \in[\mathbb{N}]^{\aleph_{0}}: k \notin x\right\}$, which is open in $[\mathbb{N}]^{\aleph_{0}}$, and similarly for $g_{k}^{-1}(-\varepsilon, \varepsilon), 0<\varepsilon<1$.

$\mathbf{0}$ is in the closure of the family $B=\left\{g_{k}: k \in \mathbb{N}\right\}$. For each $A \in \mathcal{F}$, the set $U_{A}=\left\{g \in C_{p}(\mathcal{F}):|g(A)|<1\right\}$ is a neighborhood of $\mathbf{0}$, and for each $k$ and each $A \in \mathcal{F}, k \in A$ if, and only if, $g_{k} \in U_{A}$. Consequently, for each $M \subseteq\left\{g_{k}: k \in \mathbb{N}\right\}$ and each $A \in \mathcal{F}$, we have that $M \subseteq U_{A}$ if, and only if, $\left\{k: g_{k} \in M\right\} \subseteq A$.

Assume that $B_{1}, B_{2}, \ldots$ are infinite subsets of $B=\left\{g_{k}: k \in \mathbb{N}\right\}$ as required for the Pytkeev property. Then for each $A \in \mathcal{F}$, there is $n$ such that $B_{n} \subseteq U_{A}$ and, consequently, the set $A_{n}=\left\{k: g_{k} \in B_{n}\right\}$ is a subset of $A$. The sets $A_{n}, n \in \mathbb{N}$, contradict our assumption on $\mathcal{F}$.

Lemma 8. $\mathfrak{p} \leq \mathfrak{p}_{\sigma} \leq \mathfrak{t}$.

Proof. Clearly, $\mathfrak{p} \leq \mathfrak{p}_{\sigma}$. We prove that $\mathfrak{p}_{\sigma} \leq \mathfrak{t}$.

Assume that $\left\{T_{\alpha}: \alpha<\mathfrak{t}\right\}$ is a tower with no pseudo-intersection. Given infinite sets $A_{n}, n \in \mathbb{N}$, assign to each $n$ an ordinal $\alpha_{n}<\mathfrak{t}$ such that $A_{n} \nsubseteq^{*} T_{\alpha_{n}}$. As $\mathfrak{t}$ is regular and uncountable, we have that $\alpha=\sup _{n} \alpha_{n}<\mathfrak{t}$. Then there is no $n$ such that $A_{n} \subseteq^{*} T_{\alpha}$.

Proposition 9. $\mathfrak{p}_{\sigma}=\mathfrak{p}$.

Proof. We will assume that $\mathfrak{p}<\mathfrak{p}_{\sigma}$ and show that under this assumption, $\mathfrak{p}=\mathfrak{t}$, contradicting Lemma 8.

Let $\mathcal{F} \subseteq[\mathbb{N}]^{\aleph_{0}}$ be a centered family without a pseudo-intersection, such that $|\mathcal{F}|=\mathfrak{p}$. We may assume that $\mathcal{F}$ is closed under finite intersections and enumerate it as $\mathcal{F}=\left\{A_{\alpha}: \alpha<\mathfrak{p}\right\}$.

Put $T_{0}=A_{0}, T_{1}=A_{0} \cap A_{1}, \ldots, T_{n}=\bigcap_{i \leq n} A_{i}, \ldots$, for $n \in \omega$. The permanent induction assumption is that for each $\alpha<\mathfrak{p}$, the family $\left\{T_{\beta}: \beta<\alpha\right\}$ satisfies the following:

(1) For all $\beta<\gamma<\alpha, T_{\gamma} \subseteq^{*} T_{\beta}$

(2) For all $\beta<\alpha, T_{\beta} \subseteq{ }^{*} A_{\beta}$;

(3) $\mathcal{F} \cup\left\{T_{\beta}: \beta<\alpha\right\}$ is centered.

There are three cases to consider:

$\alpha$ is a successor ordinal. If $\alpha=\beta+1$ and $T_{\beta}$ is already defined, put $T_{\alpha}=T_{\beta} \cap A_{\alpha}$. This definition and the validity of (3) for $\alpha$ imply that (1), (2) and (3) are satisfied also for $\alpha+1$.

$\alpha$ is a limit ordinal of countable cofinality. Fix an increasing sequence of ordinals $\alpha_{n}<\alpha, n \in \mathbb{N}$, such that $\sup _{n} \alpha_{n}=\alpha$, and for each $n, T_{\alpha_{n}} \backslash T_{\alpha_{n+1}}$ is infinite. (If such a choice is impossible, then there is some $\beta<\alpha$ such that for each $\gamma>\beta$, $T_{\gamma}={ }^{*} T_{\beta}$. Put $T_{\alpha}=T_{\beta} \cap A_{\alpha}$, and the situation is the same as in the successor step.) 
If there are some $A \in \mathcal{F}$ and $k$ such that $A \cap T_{\alpha_{k}}$ is a pseudo-intersection of $\left\{T_{\alpha_{n}}: n \in \mathbb{N}\right\}$, put $T_{\alpha}=A \cap A_{\alpha} \cap T_{\alpha_{k}}$. Clearly, (1), (2) and (3) again hold.

If there are no such $A \in \mathcal{F}$ and $k$, then for every $A \in \mathcal{F}$, the set $I_{A}=\{n$ : $\left.\left|A \cap\left(T_{\alpha_{n}} \backslash T_{\alpha_{n+1}}\right)\right|=\aleph_{0}\right\}$ is infinite. For each $n$, choose $T_{\alpha_{n}}^{\prime}={ }^{*} T_{\alpha_{n}}$ such that $T_{\alpha_{n}}^{\prime} \supseteq T_{\alpha_{n+1}}^{\prime}$ for all $n$. For each $A \in \mathcal{F}$, choose $f_{A} \in \mathbb{N}^{\mathbb{N}}$ such that

$$
\left|\left\{k \in A \cap\left(T_{\alpha_{n}}^{\prime} \backslash T_{\alpha_{n+1}}^{\prime}\right): k \leq f_{A}(n)\right\}\right| \geq n
$$

for all $n \in I_{A}$. Assuming $\mathfrak{p}<\mathfrak{p}_{\sigma}$, we have $\mathfrak{p}<\mathfrak{b}$ by Lemma 8 and so, having less than $\mathfrak{b}$ functions $f_{A}$, there is an upper bound $g \in \mathbb{N}^{\mathbb{N}}$. Define

$$
T_{\alpha}=A_{\alpha} \cap \bigcup_{n \in \mathbb{N}}\left\{k \in T_{\alpha_{n}}^{\prime} \backslash T_{\alpha_{n+1}}^{\prime}: k \leq g(n)\right\} .
$$

For each $n, T_{\alpha} \backslash T_{\alpha_{n}}^{\prime}$ is a finite union of finite sets, so $T_{\alpha} \subseteq^{*} T_{\alpha_{n}}^{\prime} \subseteq^{*} T_{\alpha_{n}}$. Consequently, $T_{\alpha} \subseteq^{*} T_{\beta}$ for all $\beta<\alpha$. Moreover, $T_{\alpha} \subseteq A_{\alpha}$. For each $A \in \mathcal{F}$, we have by the inequality $f_{A \cap A_{\alpha}} \leq^{*} g$ that $T_{\alpha} \cap A$ is infinite.

$\alpha$ is a limit ordinal of uncountable cofinality. Put $\kappa=\operatorname{cf}(\alpha)$ and choose a cofinal sequence $\left\langle\alpha_{\iota}: \iota<\kappa\right\rangle$ converging to $\alpha$. Let $\mathcal{F}_{\alpha}$ be the family of all intersections of finitely many elements from $\left\{T_{\alpha_{\iota}}: \iota<\kappa\right\} \cup \mathcal{F}$.

By the assumption $\mathfrak{p}<\mathfrak{p}_{\sigma}$, there is a partition $\mathcal{F}_{\alpha}=\bigcup_{n} \mathcal{F}_{n}^{\alpha}$ such that each $\mathcal{F}_{n}^{\alpha}$ has a pseudo-intersection $A_{n}$.

Let $K \subseteq \mathbb{N}$ be the set of all $n$ such that for each $\iota<\kappa$, there is $A \in \mathcal{F}_{n}^{\alpha}$ with $A \subseteq^{*} T_{\alpha_{\iota}}$. For each $n \notin K$, let $\iota_{n}<\kappa$ be such that for each $A \in \mathcal{F}_{n}^{\alpha}$ and each $\iota \geq \iota_{n}, A \nsubseteq^{*} T_{\alpha_{\iota}}$. Take $\zeta=\sup _{n \notin K} \iota_{n}$. As $\kappa$ is regular uncountable, $\zeta<\kappa$. For each $A \in \mathcal{F}_{\alpha}, T_{\alpha_{\zeta}} \cap A \notin \bigcup_{n \notin K} \mathcal{F}_{n}^{\alpha}$, and therefore there is $n \in K$ such that $T_{\alpha_{\zeta}} \cap A \in \mathcal{F}_{n}^{\alpha}$, and consequently $A_{n} \subseteq^{*} A$. Thus, we may rearrange the partition such that $\bigcup_{n \in K} \mathcal{F}_{n}^{\alpha}=\mathcal{F}_{\alpha}$. Since $\mathcal{F}$ has no pseudo-intersection and $\mathcal{F}_{\alpha}$ contains $\mathcal{F}$ and is closed under finite intersections, we have by the remark preceding Definition 4 that $K$ is infinite. Thus, we may assume that $K=\mathbb{N}$. Passing to infinite subsets, if necessary, we may assume further that the pseudo-intersections $A_{n}, n \in \mathbb{N}$, are pairwise disjoint.

For each $\iota<\kappa$, choose $f_{\iota} \in \mathbb{N}^{\mathbb{N}}$ such that for each $n$ and each $k \geq f_{\iota}(n)$ with $k \in A_{n}$, we have that $k \in T_{\alpha_{\iota}}$. Take an upper bound $g \in \mathbb{N}^{\mathbb{N}}$ for the family $\left\{f_{\iota}: \iota<\kappa\right\}$, and put $S_{\alpha}=\bigcup_{n}\left\{k \in A_{n}: k \geq g(n)\right\}$. Then the set $S_{\alpha}$ is a pseudointersection of $\left\{T_{\beta}: \beta<\alpha\right\}$, and for each $A \in \mathcal{F}$, letting $n$ be such that $A_{n} \subseteq \subseteq^{*} A$, as $A_{n} \subseteq^{*} S_{\alpha}$, we have that $S_{\alpha} \cap A$ is infinite. It remains to put $T_{\alpha}=S_{\alpha} \cap A_{\alpha}$.

Having considered all possible cases, we conclude that this inductive construction produces a tower $\left\{T_{\alpha}: \alpha<\mathfrak{p}\right\}$. But this implies that $\mathfrak{p}=\mathfrak{t}$ and consequently $\mathfrak{p}=\mathfrak{p}_{\sigma}$

This completes the proof of Theorem 3 .

Remark 10. One can define $\mathfrak{t}_{\sigma}$ to be the minimal cardinality of a $\subseteq^{*}$-linearly ordered family $\mathcal{F} \subseteq[\mathbb{N}]^{\aleph_{0}}$ such that for each partition $\mathcal{F}=\bigcup_{n \in \mathbb{N}} \mathcal{F}_{n}$, there is $n$ such that $\mathcal{F}_{n}$ has no pseudo-intersection. It was shown in Lemma 2.7 of [8] that $\mathfrak{t}=\mathfrak{t}_{\sigma}$.

The reader may wonder whether there could exist a set of reals $X$ such that $|X| \geq \mathfrak{p}$ and $C_{p}(X)$ has the Pytkeev property. The answer is positive: Recall that a space $Y$ is Fréchet if whenever $y \in \bar{A} \subseteq Y$, there is a sequence $\left\{a_{n}\right\}_{n \in \mathbb{N}}$ in $A$ such that $\lim _{n} a_{n}=y$. Clearly, every Fréchet space has the Pytkeev property. Assuming the Continuum Hypothesis (or just $\mathfrak{p}=\mathfrak{c}$ ), there is a set of reals $X$ with $|X|=\mathfrak{c}$, 
such that $C_{p}(X)$ is Fréchet [2] (such sets $X$ are usually called $\gamma$-sets [3]). We will say more about that in Theorem 18 and in the discussion following it.

\section{The Pytkeev property of $C_{p}(X,\{0,1\})$}

For a topological space $X$, and a set $S \subseteq \mathbb{R}$, let $C_{p}(X, S)$ be the collection of all continuous functions $f: X \rightarrow S$ viewed as a subspace of $C_{p}(X)$. The example in Lemma 7 actually shows that there is a set $X \subseteq \mathbb{R}$ of cardinality $\mathfrak{p}_{\sigma}$ such that $C_{p}(X,\{0,1\})$ does not have the Pytkeev property. This motivates the following theorem.

For a centered family $\mathcal{F} \subseteq[\mathbb{N}]^{\aleph_{0}}$, denote by $\langle\mathcal{F}\rangle$ the closure of $\mathcal{F}$ under intersections of finite subsets. Say that $\mathcal{F}$ is free if $\bigcap \mathcal{F}=\emptyset$. Recall that $\mathcal{U}$ is an $\omega$-cover of a space $X$ if $X \notin \mathcal{U}$, but each finite $F \subseteq X$ is contained in some $U \in \mathcal{U}$.

Theorem 11. The following are equivalent:

(1) $C_{p}(X,\{0,1\})$ has the Pytkeev property.

(2) Each clopen $\omega$-cover of $X$ contains a countable $\omega$-cover of $X$; and For each continuous free centered image $\mathcal{F}$ of $X$ in $[\mathbb{N}]^{\aleph_{0}}$, there is a partition $\langle\mathcal{F}\rangle=\bigcup_{n} \mathcal{F}_{n}$ such that each $\mathcal{F}_{n}$ has a pseudo-intersection.

(3) Each clopen $\omega$-cover $\mathcal{U}$ of $X$ contains infinite subsets $\mathcal{U}_{1}, \mathcal{U}_{2}, \ldots$, such that $\left\{\bigcap \mathcal{U}_{n}: n \in \mathbb{N}\right\}$ is an $\omega$-cover of $X$.

Proof. $(1 \Rightarrow 3)$ Assume that $\mathcal{U}$ is a clopen $\omega$-cover of $X$. Let $A=\left\{\chi_{U}: U \in \mathcal{U}\right\}$. Then $\mathbf{1} \in \bar{A} \backslash A$, where $\mathbf{1}$ is the function constantly equal to 1 . Use (1) to obtain infinite subsets $A_{1}, A_{2}, \ldots$ of $A$ as in the definition of the Pytkeev property. For each $n$, let $\mathcal{U}_{n}=\left\{U: \chi_{U} \in A_{n}\right\}$.

Fix a finite set $F \subseteq X$. As $U_{F}=\left\{f \in C_{p}(X,\{0,1\}): f \uparrow F \equiv 1\right\}$ is a neighborhood of $\mathbf{1}$, there is $n$ such that $A_{n} \subseteq U_{F}$. For each $U \in \mathcal{U}_{n}, \chi_{U} \in A_{n} \subseteq U_{F}$, and therefore $F \subseteq U$. This shows that $F \subseteq \bigcap \mathcal{U}_{n}$.

$(3 \Rightarrow 1)$ As $C_{p}(X,\{0,1\})$ is homogeneous, it suffices to prove that it has the Pytkeev property at $\mathbf{1}$. Assume that $A \subseteq X$ and $\mathbf{1} \in \bar{A} \backslash A \subseteq C_{p}(X,\{0,1\})$. Then $\mathcal{U}=\left\{g^{-1}(1): g \in A\right\}$ is a clopen $\omega$-cover of $X$. Choose infinite subsets $\mathcal{U}_{n}$ of $\mathcal{U}$ such that $\left\{\bigcap \mathcal{U}_{n}: n \in \mathbb{N}\right\}$ is an $\omega$-cover of $X$, and for each $n$ take $A_{n}=\{g \in A$ : $\left.g^{-1}(1) \in \mathcal{U}_{n}\right\}$.

For each neighborhood $U$ of $\mathbf{1}$, take a finite $F \subseteq X$ such that $U_{F}=\{f \in$ $\left.C_{p}(X,\{0,1\}): f \uparrow F \equiv 1\right\} \subseteq U$. Choose $n$ such that $F \subseteq \bigcap \mathcal{U}_{n}$. For each $g \in A_{n}$, $g^{-1}(1) \in \mathcal{U}_{n}$, and therefore $g \uparrow F \equiv 1$. Thus, $g \in U_{F} \subseteq U$. This shows that $A_{n} \subseteq U_{F} \subseteq U$.

$(2 \Rightarrow 3)$ Assume that $\mathcal{U}$ is a clopen $\omega$-cover of $X$. Choose a countable $\omega$ subcover $\mathcal{V} \subseteq \mathcal{U}$ of $X$. Enumerate $\mathcal{V}=\left\{U_{n}: n \in \mathbb{N}\right\}$ bijectively, and define $\Psi: X \rightarrow[\mathbb{N}]^{\aleph_{0}}$ by $\Psi(x)=\left\{n: x \in U_{n}\right\}$. As $\mathcal{V}$ is an $\omega$-cover of $X, \Psi[X] \subseteq[\mathbb{N}]^{\aleph_{0}}$, and is free and centered. As the sets $U_{n}$ are clopen, $\Psi$ is continuous. Take a partition $\langle\Psi[X]\rangle=\bigcup_{n} \mathcal{F}_{n}$ such that $\bigcap \mathcal{F}_{n}$ is infinite for each $n$ (recall Remark 5). For each $n$, take $\mathcal{U}_{n}=\left\{U_{m}: m \in \bigcap \mathcal{F}_{n}\right\}$.

Assume that $F \subseteq X$ is finite. Take $n$ such that

$$
A=\left\{n: F \subseteq U_{n}\right\}=\bigcap_{x \in F}\left\{n: x \in U_{n}\right\}=\bigcap_{x \in F} \Psi(x) \in \mathcal{F}_{n} .
$$

For each $U_{m} \in \mathcal{U}_{n}, m \in \bigcap \mathcal{F}_{n} \subseteq A$, and therefore $F \subseteq U_{m}$. It follows that $F \subseteq \bigcap \mathcal{U}_{n}$. 
$(3 \Rightarrow 2)$ Our assumption implies that every clopen $\omega$-cover of $X$ contains a countable $\omega$-cover of $X$ : If $\left\{\bigcap \mathcal{U}_{n}: n \in \mathbb{N}\right\}$ is an $\omega$-cover of $X$ and we choose for each $n$ an element $U_{n} \in \mathcal{U}_{n}$, then $\left\{U_{n}: n \in \mathbb{N}\right\}$ is an $\omega$-cover of $X$.

Now, assume that $\Psi: X \rightarrow[\mathbb{N}]^{\aleph_{0}}$ is continuous and its image $\mathcal{F}$ is free and centered. Setting $U_{n}=\{x \in X: n \in \Psi(x)\}$ for each $n$, we have that $\mathcal{U}=\left\{U_{n}\right.$ : $n \in \mathbb{N}\}$ is a clopen $\omega$-cover of $X$. Let $\mathcal{U}_{m}, m \in \mathbb{N}$, be infinite subsets of $\mathcal{U}$ such that $\left\{\bigcap \mathcal{U}_{m}: m \in \mathbb{N}\right\}$ is an $\omega$-cover of $X$, and take $A_{m}=\left\{n: U_{n} \in \mathcal{U}_{m}\right\}$ for each $m$. Each $A \in\langle\mathcal{F}\rangle$ has the form $A=\bigcap_{x \in F} \Psi(x)$ for a finite $F \subseteq X$, and if $m$ is such that $F \subseteq \bigcap \mathcal{U}_{m}$, then for each $n \in A_{m}, F \subseteq U_{n}$, and therefore $n \in A$. This shows that for each $A \in\langle\mathcal{F}\rangle$ there is $m$ such that $A_{m} \subseteq A$. Clearly, this suffices.

The first assumption in item (2) of Theorem 11 is a property of $C_{p}(X,\{0,1\})$.

Lemma 12 (Folklore). The following are equivalent:

(1) $C_{p}(X,\{0,1\})$ has countable tightness;

(2) Each clopen $\omega$-cover of $X$ contains a countable $\omega$-cover of $X$.

Proof. $(1 \Rightarrow 2)$ Assume that $\mathcal{U}$ is a clopen $\omega$-cover of $X$. Let $A=\left\{\chi_{U}: U \in \mathcal{U}\right\}$. Then $\mathbf{1} \in \bar{A} \backslash A$. As $C_{p}(X,\{0,1\})$ has countable tightness, there is a countable set $\mathcal{V}=\left\{U_{n}: n \in \mathbb{N}\right\} \subseteq \mathcal{U}$ such that $\mathbf{1} \in \overline{\left\{\chi_{U_{n}}: n \in \mathbb{N}\right\}}$. It follows that $\left\{U_{n}: n \in \mathbb{N}\right\}$ is an $\omega$-cover of $X$.

$(2 \Rightarrow 1)$ If $\mathbf{1} \in \bar{A} \backslash A \subseteq C_{p}(X,\{0,1\})$, then $\mathcal{U}=\left\{g^{-1}(1): g \in A\right\}$ is a clopen $\omega$-cover of $X$, and taking a countable subset $\left\{g_{n}^{-1}(1): n \in \mathbb{N}\right\}$ of $\mathcal{U}$ which is an $\omega$-cover of $X$, we have that 1 is in the closure of $\left\{g_{n}: n \in \mathbb{N}\right\}$.

This will be used later in this paper.

\section{From $C_{p}(X,\{0,1\})$ BaCK то $C_{p}(X)$}

The following basic observation will be used in order to get back to $C_{p}(X)$. Let

$$
S_{0}=\left\{\frac{1}{n}: n \in \mathbb{N}\right\} \cup\{0\} .
$$

Our route to $C_{p}(X)$ goes via $C_{p}\left(X, S_{0}\right)$.

Lemma 13 (Discretization). Assume that $X$ is Lindelöf and zero-dimensional. Then there is a function $D: C_{p}(X, \mathbb{R}) \rightarrow C_{p}\left(X, S_{0}\right)$ such that for each $x \in X$ :

(1) $f(x)=0$ if, and only if, $D(f)(x)=0$;

(2) For each $k \geq 2$

(a) if $|f(x)| \leq \frac{1}{k}$, then $D(f)(x) \leq \frac{1}{k}$;

(b) if $D(f)(x)<\frac{1}{k}$, then $|f(x)|<\frac{1}{k}$.

Proof. As $X$ is Lindelöf and zero-dimensional, it has large inductive dimension zero, and consequently has the property that zero sets are separated by clopen sets; that is, for all disjoint zero sets (preimages of 0 under continuous real-valued functions on $X) A, B \subseteq X$, there is a clopen $C \subseteq X$ such that $A \subseteq C$ and $C \cap B=\emptyset$. We will only assume this latter property in our proof.

Fix $f \in C_{p}(X)$. For each $n,\left[0, \frac{1}{n+1}\right]$ and $\left[\frac{1}{n}, \infty\right)$ are closed in $\mathbb{R}$ and are therefore zero sets. Thus, $|f|^{-1}\left[0, \frac{1}{n+1}\right]$ and $|f|^{-1}\left[\frac{1}{n}, \infty\right)$ are (disjoint) zero sets in $X$. Take a clopen $C_{n} \subseteq X$ such that $|f|^{-1}\left[\frac{1}{n}, \infty\right) \subseteq C_{n}$ and $C_{n} \cap|f|^{-1}\left[0, \frac{1}{n+1}\right]=\emptyset$. For each $n, C_{n-1} \subseteq X \backslash|f|^{-1}\left[0, \frac{1}{n}\right]=|f|^{-1}\left(\frac{1}{n}, \infty\right) \subseteq C_{n}$. Define $V_{n}=C_{n} \backslash C_{n-1}$. Then $\bigcup_{n} V_{n}=X \backslash f^{-1}(0)$. 
For each $x \in X \backslash f^{-1}(0)$, there is a unique $n$ with $x \in V_{n}$. Define $D(f)(x)=\frac{1}{n}$. If $x \in f^{-1}(0)$, define $D(f)(x)=0$. It is not difficult to verify that $D(f)$ is continuous.

$D$ has the required properties: $f(x)=0$ if, and only if, $D(f)(x)=0$. Assume that $f(x) \neq 0$ and $|f(x)| \leq \frac{1}{k}$. Let $n$ be such that

$$
\frac{1}{n+1}<|f(x)| \leq \frac{1}{n}
$$

Necessarily, $k \leq n$, and $x \in C_{n+1} \backslash C_{n-1}$. Thus, $D(f)(x) \in\left\{\frac{1}{n+1}, \frac{1}{n}\right\}$, and therefore $D(f)(x) \leq \frac{1}{n} \leq \frac{1}{k}$.

On the other hand, if $D(f)(x)<\frac{1}{k}$, let $n>k$ be such that $|D(f)(x)|=\frac{1}{n}$. Then $x \in V_{n}$, and therefore

$$
|f(x)|<\frac{1}{n-1} \leq \frac{1}{k}
$$

To move from $C_{p}(X,\{0,1\})$ to $C_{p}\left(X, S_{0}\right)$, we will need the following.

Lemma 14 (Finitization). Fix any topological space $X$. For each $k$, there is a function $\Phi_{k}: C_{p}\left(X, S_{0}\right) \rightarrow C_{p}(X,\{0,1\})$ such that for each $x \in X:|f(x)|<\frac{1}{k}$ if, and only if, $\Phi_{k}(f)(x)=0$.

Proof. The assertion in the lemma defines $\Phi_{k}$, which is clearly continuous.

Theorem 15. The following are equivalent for Tychonoff spaces $X$ :

(1) $C_{p}(X)$ has the Pytkeev property.

(2) $X$ is zero-dimensional, and $C_{p}\left(X, S_{0}\right)$ has the Pytkeev property.

(3) $X$ is zero-dimensional, and $C_{p}(X,\{0,1\})$ has the Pytkeev property.

Proof. ( $1 \Rightarrow 2 \Rightarrow 3)$ Recall that for Tychonoff spaces, (1) implies that $X$ is zerodimensional [5]. To obtain the Pytkeev property, use the inclusions $C_{p}(X,\{0,1\}) \subseteq$ $C_{p}\left(X, S_{0}\right) \subseteq C_{p}(X)$ of topological spaces.

$(3 \Rightarrow 1)$ Assume that $(3)$ holds. We first prove that $C_{p}\left(X, S_{0}\right)$ has the Pytkeev property at $\mathbf{0}$. Assume that $A \subseteq C_{p}\left(X, S_{0}\right)$ and $\mathbf{0} \in \bar{A} \backslash A$.

Case 1. For an infinite set $I$ of natural numbers $k$, there is $f_{k} \in A$ with $\left|f_{k}(x)\right|<1 / k$ for all $x \in X$. Then $f_{k} \rightarrow \mathbf{0}$, and the sets $\left\{f_{k}: n \leq k \in I\right\}, n \in \mathbb{N}$, are as required.

Case 2. There is $K$ such that for all $k \geq K$ and each $f \in A$, there is $x \in X$ with $|f(x)| \geq 1 / k$. Then for each $k \geq K, \mathbf{0} \notin \Phi_{k}[A]$.

Fix $k \geq K$. By Lemma 14, $\Phi_{k}[A] \subseteq C_{p}(X,\{0,1\})$ and $\mathbf{0} \in \overline{\Phi_{k}[A]} \backslash \Phi_{k}[A]$. By (3), there are sets $A_{n}^{k} \subseteq A, n \in \mathbb{N}$, such that the sets $\Phi_{k}\left[A_{n}^{k}\right]$ are infinite and each neighborhood of $\mathbf{0}$ contains one of them.

Given a neighborhood $\left[x_{1}, \ldots, x_{m} ; k\right]$ of $\mathbf{0}$ where $k \geq K$, let $n$ be such that $\Phi_{k}\left[A_{n}^{k}\right] \subseteq\left[x_{1}, \ldots, x_{m} ; 2\right]$. By Lemma $14, A_{n}^{k} \subseteq\left[x_{1}, \ldots, x_{m} ; k\right]$. Thus, the countable family $\left\{A_{n}^{k}: k, n \in \mathbb{N}, k \geq K\right\}$ of subsets of $A$ witnesses the Pytkeev property of $C_{p}\left(X, S_{0}\right)$ at $\mathbf{0 .}$

We can now prove that (1) holds. As the Pytkeev property implies countable tightness, we have by Lemma 12 that each clopen $\omega$-cover of $X$ contains a countable cover of $X$. It follows that $X$ is Lindelöf: given an open cover $\mathcal{U}$ of $X$ which does not contain a finite subcover, use the zero-dimensionality of $X$ to replace $\mathcal{U}$ by all finite unions of clopen sets contained in members of $\mathcal{U}$. We obtain a clopen $\omega$-cover of $X$; take a countable subcover of it. Thus, Lemma 13 is applicable to $X$. 
Assume that $A \subseteq C_{p}(X)$ and $\mathbf{0} \in \bar{A} \backslash A$. By Lemma 13, $D[A] \subseteq C_{p}\left(X, S_{0}\right)$ and $\mathbf{0} \in \overline{D[A]} \backslash D[A]$. By the Pytkeev property of $C_{p}\left(X, S_{0}\right)$ at $\mathbf{0}$, there are sets $A_{n} \subseteq A, n \in \mathbb{N}$, such that the sets $D\left[A_{n}\right]$ are infinite (subsets of $D[A]$ ), and each neighborhood of $\mathbf{0}$ contains one of the sets $D\left[A_{n}\right]$.

Given a neighborhood $\left[x_{1}, \ldots, x_{m} ; k\right]$ of $\mathbf{0}$, let $n$ be such that $D\left[A_{n}\right]$ is a subset of this neighborhood. By Lemma 13, $A_{n} \subseteq\left[x_{1}, \ldots, x_{m} ; k\right]$. The sets $A_{n} \subseteq A$ are infinite since the sets $D\left[A_{n}\right]$ are. This shows that $C_{p}(X)$ has the Pytkeev property at $\mathbf{0}$. As $C_{p}(X)$ is a topological group, this suffices.

Remark 16. We cannot remove the assumption of zero-dimensionality from (2) and (3) of Theorem 15: Let $X=\mathbb{Q} \times \mathbb{R} . \quad X$ is Lindelöf. As $X$ is not zerodimensional, $C_{p}(X)$ does not have the Pytkeev property. Since $\mathbb{R}$ is connected, any $f \in C_{p}(\mathbb{Q} \times \mathbb{R}, Y)$, where $Y$ is zero-dimensional, is constant on each fiber $\{q\} \times \mathbb{R}, q \in \mathbb{Q}$. It follows that $C_{p}(X,\{0,1\})$ is homeomorphic to $C_{p}(\mathbb{Q},\{0,1\})$, and $C_{p}\left(X, S_{0}\right)$ is homeomorphic to $C_{p}\left(\mathbb{Q}, S_{0}\right)$. As $\mathbb{Q}$ is countable, these spaces both have the Pytkeev property.

Corollary 17. The following are equivalent for zero-dimensional spaces $X$ :

(1) $C_{p}(X)$ has the Pytkeev property.

(2) Each clopen $\omega$-cover of $X$ contains a countable $\omega$-cover of $X$; and For each continuous free centered image $\mathcal{F}$ of $X$ in $[\mathbb{N}]^{\aleph_{0}}$, there is a partition $\langle\mathcal{F}\rangle=\bigcup_{n} \mathcal{F}_{n}$ such that each $\mathcal{F}_{n}$ has a pseudo-intersection.

(3) Each clopen $\omega$-cover $\mathcal{U}$ of $X$ contains infinite subsets $\mathcal{U}_{1}, \mathcal{U}_{2}, \ldots$, such that $\left\{\bigcap \mathcal{U}_{n}: n \in \mathbb{N}\right\}$ is an $\omega$-cover of $X$.

Proof. Theorems 11 and 15.

A problem of Sakai [5, Question 1] would be solved if "clopen" could be replaced by "open" in (3) of Corollary 17. This is even interesting for $X \subseteq \mathbb{R} \backslash \mathbb{Q}$.

Another open problem is whether, for each set of reals $X$, if $C_{p}(X)$ has the Pytkeev property, then $C_{p}(X)$ is Fréchet [7]. Recall that the converse implication holds.

According to Borel, a metric space $X$ has strong measure zero if for each sequence of positive reals $\left\{\epsilon_{n}\right\}_{n \in \mathbb{N}}$, there exists a cover $\left\{I_{n}\right\}_{n \in \mathbb{N}}$ of $X$ by open balls, such that for each $n$, the diameter of $I_{n}$ is smaller than $\epsilon_{n}$. If $X$ is a set of reals and $C_{p}(X)$ is Fréchet, then $X$ has strong measure zero [3]. Answering a question of ours, Miller proved the following.

Theorem 18 (Miller). If $X \subseteq \mathbb{R}$ and $C_{p}(X)$ has the Pytkeev property, then $X$ has strong measure zero.

Proof. By standard arguments, we may assume that $X \subseteq\{0,1\}^{\mathbb{N}}[10] .^{1}$ It suffices to prove that for each increasing sequence $\left\{k_{n}\right\}_{n \in \mathbb{N}}$ of natural numbers, there are for each $n$ elements $s_{m}^{n} \in\{0,1\}^{k_{n}}, m \leq n$, such that $X=\bigcup_{n}\left(\left[s_{1}^{n}\right] \cup \cdots \cup\left[s_{n}^{n}\right]\right)$. (One can allow $n$ sets of diameter $\epsilon_{n}$ in the original definition of strong measure zero by moving to an appropriate subsequence of the original sequence $\left\{\epsilon_{n}\right\}_{n \in \mathbb{N}}[10]$.)

\footnotetext{
${ }^{1}$ For each $n, X \cap[-n, n]$ is a closed subset of $X$ and therefore $C_{p}(X \cap[-n, n])$ has the Pytkeev property; e.g., by Corollary $17(3)$. The mapping $T:\{0,1\}^{\mathbb{N}} \rightarrow[0,1]$, defined by $x \mapsto \sum_{i=1}^{\infty} x(i) / 2^{i}$, preserves strong measure zero in both directions [10], so we can transform $X \cap[-n, n]$ into $\{0,1\}^{\mathbb{N}}$ and prove - as is done later in this paper - that it has strong measure zero. As $X=\bigcup_{n} X \cap[-n, n]$ is a countable union, it will follow that $X$ has strong measure zero.
} 
For each $n$, let

$$
\mathcal{U}_{n}=\left\{\left[s_{1}\right] \cup \cdots \cup\left[s_{n}\right]: s_{1}, \ldots, s_{n} \in\{0,1\}^{k_{n}}\right\},
$$

and take $\mathcal{U}=\bigcup_{n} \mathcal{U}_{n} . \mathcal{U}$ is a clopen $\omega$-cover of $X$. By Corollary 17, there are infinite subsets $\mathcal{V}_{1}, \mathcal{V}_{2}, \ldots$ of $\mathcal{U}$, such that $\left\{\bigcap \mathcal{V}_{n}: n \in \mathbb{N}\right\}$ is an $\omega$-cover of $X$. As each $\mathcal{V}_{n}$ is infinite and each $\mathcal{U}_{n}$ is finite, we can find $m_{1}$ and $V_{1} \in \mathcal{V}_{1} \cap \mathcal{U}_{m_{1}}, m_{2}>m_{1}$ and $V_{2} \in \mathcal{V}_{2} \cap \mathcal{U}_{m_{2}}$, etc. Then $\left\{V_{n}: n \in \mathbb{N}\right\}$ is an $\omega$-cover (in particular, a cover) of $X$, and the sets $V_{n}$ are as required in the first paragraph of this proof.

By a classical result of Laver, it is consistent that there are no uncountable strong measure zero sets. It follows that, consistently, for each uncountable $X \subseteq \mathbb{R}, C_{p}(X)$ does not have the Pytkeev property.

\section{An Application to the ReZnichenko property}

The approach of Section 4 may be useful for other local properties. We demonstrate this for the Reznichenko property.

A space $Y$ has the Reznichenko property (or is weakly Fréchet-Urysohn) if, for each $A \subseteq Y$ and each $y \in \bar{A} \backslash A$, there are pairwise disjoint finite sets $F_{n} \subseteq A$, $n \in \mathbb{N}$, such that each neighborhood of $y$ intersects all but finitely many of the sets $F_{n}$.

Clearly, the Pytkeev property implies the Reznichenko property, which in turn implies countable tightness. See [7] for a survey concerning the Reznichenko property.

A cover $\mathcal{U}$ of a space $X$ is $\omega$-groupable if there is a partition $\mathcal{P}$ of $\mathcal{U}$ into finite pieces such that for each finite $F \subseteq X$ and all but finitely many $\mathcal{F} \in \mathcal{P}, F$ is contained in some member of $\mathcal{F}$. Note that for each countable $\mathcal{C} \subseteq \mathcal{P}, \cup \mathcal{C} \subseteq \mathcal{U}$ is countable and is $\omega$-groupable, as is witnessed by its partition $\mathcal{C}$. Restricting attention to countable $\omega$-groupable covers brings us to the following [9]. A (nonprincipal) filter $\mathcal{F}$ on $\mathbb{N}$ is feeble if there is an increasing $h \in \mathbb{N}^{\mathbb{N}}$ such that each $a \in \mathcal{F}$ intersects all but finitely many of the sets $[h(n), h(n+1)), n \in \mathbb{N}$.

The second author proved that if $C_{p}(X)$ has the Reznichenko property, then no continuous image of $X$ in $[\mathbb{N}]^{\aleph_{0}}$ is a subbase for a nonfeeble filter [9]. We can now show that the converse implication also holds.

Theorem 19. The following are equivalent for zero-dimensional spaces $X$ :

(1) $C_{p}(X)$ has the Reznichenko property.

(2) Each clopen $\omega$-cover of $X$ contains a countable $\omega$-cover of $X$; and No continuous image of $X$ in $[\mathbb{N}]^{\aleph_{0}}$ is a subbase for a nonfeeble filter.

(3) Each clopen $\omega$-cover of $X$ contains an $\omega$-groupable cover of $X$.

Proof. $(2 \Leftrightarrow 3)$ is proved in [9].

$(1 \Rightarrow 3)$ is proved in [5]. (For an easier proof, note that (1) implies that the subspace $C_{p}(X,\{0,1\})$ of $C_{p}(X)$ has the Reznicenko property, and use the natural translation arguments [6].)

$(3 \Rightarrow 1)$ By $(3), C_{p}(X,\{0,1\})$ has the Reznichenko property [6]. By a general result of Sakai [6], we get that $C_{p}\left(X, S_{0}\right)$ has the Reznichenko property at $\mathbf{0}$. For completeness, we give a slightly more direct proof of this assertion.

Assume that $A \subseteq C_{p}\left(X, S_{0}\right)$ and $\mathbf{0} \in \bar{A} \backslash A$. If for an infinite set $I$ of natural numbers $n$, there is $f_{n} \in A$ with $\left|f_{n}(x)\right|<1 / k$ for all $x \in X$, then $f_{n} \rightarrow \mathbf{0}$, and 
we are done. Otherwise, let $K$ be such that for all $k \geq K$ and each $f \in A$, there is $x \in X$ with $|f(x)| \geq 1 / k$. Then for each $k \geq K, \mathbf{0} \notin \Phi_{k}[A]$.

Fix $k \geq K$. By Lemma 14, $\Phi_{k}[A] \subseteq C_{p}(X,\{0,1\})$ and $\mathbf{0} \in \overline{\Phi_{k}[A]} \backslash \Phi_{k}[A]$. By the Reznichenko property of $C_{p}(X,\{0,1\})$, there are pairwise disjoint finite sets $F_{n}^{k} \subseteq A, n \in \mathbb{N}$, such that the sets $\Phi_{k}\left[F_{n}^{k}\right]$ eventually intersect each neighborhood of $\mathbf{0}$. Given a neighborhood $\left[x_{1}, \ldots, x_{m} ; k\right]$ of $\mathbf{0}$ where $k \geq K$ and large enough $n$, $\Phi_{k}\left[F_{n}^{k}\right] \cap\left[x_{1}, \ldots, x_{m} ; 2\right] \neq \emptyset$. By Lemma $14, F_{n}^{k} \cap\left[x_{1}, \ldots, x_{m} ; k\right] \neq \emptyset$.

Define an increasing sequence $m_{n}, n \geq K$, inductively as follows. Set $m_{K}=1$ and $P_{K}=F_{1}^{K}$. Assume, inductively, that $n>k$ and the sets $P_{K}, \ldots, P_{n-1}$ are finite and disjoint. For each $k=K, \ldots, n$ the disjointness of the sets $F_{i}^{k}, i \in \mathbb{N}$, allows us to find $i_{k}>m_{n}$ such that for each $i \geq i_{k}, F_{i}^{k}$ is disjoint from $P_{K} \cup \cdots \cup P_{n-1}$. Set $m_{n}=\max \left\{i_{1}, \ldots, i_{n}\right\}$, and take $P_{n+1}=\bigcup_{k=K}^{n} F_{m_{n}}^{k}$.

Fix a neighborhood $\left[x_{1}, \ldots, x_{m} ; k\right]$ of $\mathbf{0}$. For all large enough $n$ (so that in particular, $n>k), F_{m_{n}}^{k}$ intersects $\left[x_{1}, \ldots, x_{m} ; k\right]$. As $F_{m_{n}}^{k} \subseteq P_{n}$, the sets $P_{n}$, $n \in \mathbb{N}$, witness the Reznichenko property of $C_{p}\left(X, S_{0}\right)$ at $\mathbf{0}$.

We can now prove that $C_{p}(X)$ has the Reznichenko property. Assume that $A \subseteq C_{p}(X)$, and $\mathbf{0} \in \bar{A} \backslash A$. By Lemma $13, D[A] \subseteq C_{p}\left(X, S_{0}\right)$ and $\mathbf{0} \in \overline{D[A]} \backslash D[A]$. By (2), there are finite $F_{n} \subseteq A$ such that each neighborhood of $\mathbf{0}$ intersects $D\left[F_{n}\right]$ for all but finitely many $n$. By Lemma 13 again, each neighborhood of $\mathbf{0}$ intersects $F_{n}$ for all but finitely many $n$.

Here, too, a problem of Sakai [5, Question 4] would be solved if "clopen" could be replaced by "open" in (2) of Theorem 19. In any case, the characterizations given in Corollary 17 and in Theorem 19 for the Pytkeev and Reznichenko properties of $C_{p}(X)$, respectively, are more natural than the other known characterizations (see $[5])$.

\section{ACKNOWLEDGMENTS}

We thank Masami Sakai, Nadav Samet, and Lyubomyr Zdomskyy for their useful comments on the paper. We also thank Arnold Miller for Theorem 18.

\section{REFERENCES}

[1] A. R. Blass, Combinatorial cardinal characteristics of the continuum, in: Handbook of Set Theory (M. Foreman, A. Kanamori, and M. Magidor, eds.), Kluwer Academic Publishers, Dordrecht, to appear. http://www.math.lsa.umich.edu/ ablass/hbk.pdf

[2] F. Galvin and A. W. Miller, $\gamma$-sets and other singular sets of real numbers, Topology and its Applications 17 (1984), 145-155. MR738943 (85f:54011)

[3] J. Gerlits and Zs. Nagy, Some properties of $C(X)$, I, Topology and its Applications 14 (1982), 151-161. MR667661 (84f:54021)

[4] E. G. Pytkeev, On maximally resolvable spaces, Proceedings of the Steklov Institute of Mathematics 154 (1984), 225-230.

[5] M. Sakai, The Pytkeev property and the Reznichenko property in function spaces, Note di Matematica 22 (2003), 43-52. MR2112730 (2005i:54020)

[6] M. Sakai, Two properties of $C_{p}(X)$ weaker than the Fréchet Urysohn property, Topology and its Applications 153 (2006), 2795-2804 . MR2248383 (2007c:54004)

[7] M. Sakai, Special subsets of reals characterizing local properties of function spaces, in: Selection Principles and Covering Properties in Topology (Lj. D.R. Kočinac, ed.), Quaderni di Matematica, to appear.

[8] B. Tsaban, A topological interpretation of $\mathfrak{t}$, Real Analysis Exchange 25 (1999/2000), 391404. MR1691758 (2000j:03066)

[9] B. Tsaban, The minimal cardinality where the Reznichenko property fails, Israel Journal of Mathematics 140 (2004), 367-374. MR2054852 (2005g:54008) 
[10] B. Tsaban and T. Weiss, Products of special sets of real numbers, Real Analysis Exchange $30(2004 / 5)$, 819-836. MR2177439 (2006g:26005)

Department of Computer Science and Mathematical Logic, Charles University, Malostranské nám. 25, 11000 Praha 1, CZech Republic.

E-mail address: psimon@ms.mff.cuni.cz

Department of Mathematics, Bar-Ilan University, Ramat-Gan 52900, Israel; and Department of Mathematics, Weizmann Institute of Science, Rehovot 76100, Israel

E-mail address: tsaban@math.biu.ac.il

URL: http://www.cs.biu.ac.il/ ${ }^{\text {tsaban }}$ 